\title{
Graphical Approach to Index and Retrieve Standard Young Tableaux
}

\author{
A. Ganapathi Rao \\ Dept. of Mathematics, GMRIT, Rajam, Srikakulam
}

\author{
N. Ravi Shankar \\ Dept. of Applied Mathematics, GIS, GITAM \\ University, Visakhapatnam.
}

\begin{abstract}
The Standard Young Tableaux are used to label the basis vectors of the standard or Young Yamanouchi basis of the symmetric group. There is a one - to - one correspondence between the eigen values of the Complete Set of Commuting Operators-I (CSCO-I) of Symmetric group of degree $n\left(S_{n}\right)$ and the Standard Young Tableaux(SYT). In this paper, a new graphical method to index and retrieve the standard Young tableau for a partition of degree $n$ in $S_{n}$ is presented. This method is illustrated with an example using a partition of degree 6 in symmetric group $\mathrm{S}_{6}$.
\end{abstract}

\section{Keywords:}

Symmetric group; Standard Young Tableaux; indexing; retrieving; CSCO-I

\section{INTRODUCTION}

In $[3,6]$, the Young tableaux provide a convenient method of determining the weight vectors of a given irreducible representation of the classical group and the corresponding weight multiplicities. Stanley [1], motivated by the work of Patera and Sharp [2], demonstrated that the standard Young tableaux of classical group have an important role to play in determining the character generator of that classical group. Combinatorial items like graphs and trees in Graph theory, partitions in set theory, Standard Young Tableaux (SYT) and Semi Standard Young Tableaux (SSYT) in group theory occur naturally in Science and Engineering. In many situations, we need to index and retrieve these combinatorial items. Wilf [4] developed an algorithm to rank and unrank combinatorial items. It is also known that there is a one - to - one correspondence between the eigen values of the Complete Set of Commuting Operators-I (CSCO-I) of Symmetric group of degree $n\left(S_{n}\right)$ and the SYT. In this paper, we show how this result and the general framework given by Wilf [4] can be adapted to index and retrieve SYT. A new graphical method is introduced to index and retrieve the SYT for a partition of degree $\mathrm{n}$ in symmetric group using branching rule.

\section{PRELIMINARIES AND BRANCHING LAW}

Definition 1. A Partition of a positive integer $\mathrm{n}$ is a sequence of positive integers $\lambda=\left(\lambda_{1}, \lambda_{2}, \ldots, \lambda_{\mathrm{k}}\right)$ satisfying $\lambda_{1} \geq \lambda_{2} \geq$ $\ldots \geq \lambda_{\mathrm{k}}>0$ and $\mathrm{n}=\lambda_{1}+\lambda_{2}+\ldots+\lambda_{\mathrm{k}}$.

For instance, the number 5 has seven partitions : $(5),(4,1),(3,2)$, $(3,1,1),(2,2,1),(2,1,1,1),(1,1,1,1,1)$

Definition 2. A Young diagram or Ferrers diagram is a finite collection of boxes arranged in left-justified rows, with the row sizes weakly decreasing.

The Young diagram associated to the partition $\lambda=\left(\lambda_{1}, \lambda_{2}\right.$, $\left.\ldots, \lambda_{\mathrm{k}}\right)$ is the one that has $\mathrm{k}$ rows, and $\lambda_{\mathrm{i}}$ boxes on the $\mathrm{i}^{\text {th }}$ row.

For instance, the Young diagrams corresponding to the partitions of 5 are

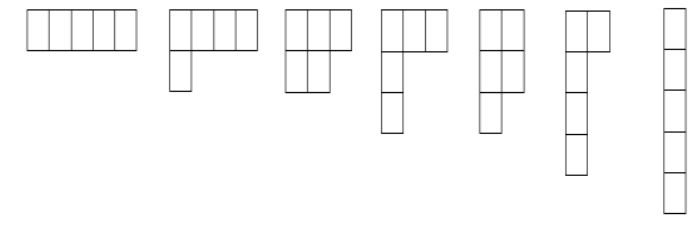

(5) $\quad(4,1) \quad(3,2) \quad(3,1,1) \quad(2,2,1) \quad(2,1,1,1) \quad(1,1,1,1,1)$

Definition 3. A (Young) tableau $t$ of shape $\lambda$, is obtained by filling in the boxes of a young diagram of $\lambda$ with 1,2 , $3 \ldots \ldots, n$, with each number occurring exactly once. in this case, we say that $\mathrm{t}$ is a $\lambda$-tableau.

Definition 4. A standard (Young) tableau (SYT) is a young tableau whose the entries are increasing across each row and each column.

The permutation group has important applications in physics for many particle systems. The importance lies in the fact that a system of identical particles has permutation symmetry and that there exist many deep and delicate inter relations between the group and the unitary group. The representation theory of the permutation group is well established through the effect of Young, Frobenious, and Yamanouchi and other. This representation theory has many advantages, for example it gives the branching law for reducing the irreducible representation of $S_{n}$, into those of $S_{n-1}$, the intuitive and elegant way of labeling the irreducible way representation and the irreducible bases by the Young diagrams and the Young tableaux, etc.

Branching law:

An irreducible representation $[v]$ of $S_{n}$ is an general reducible with respect to its subgroup $\mathrm{S}_{\mathrm{n}-1}$, $[v] \downarrow S_{n-1}=\sum_{v^{\prime}} \oplus D^{\left[v^{\prime}\right]}\left(S_{n-1}\right)$, where the Young diagram $\left[v^{\prime}\right]$ result from removing one box in all possible way in the young diagram $[v]$, and each irreducible representation $\left[v^{\prime}\right]$ occurs only once. For example the irreducible representation [ [ $\left.\begin{array}{lll}4 & 3 & 1\end{array}\right]$ of $S_{8}$ contains each of the irreducible representation [4 3], [4 2 1] and [ [ $\left.\begin{array}{lll}3 & 3 & 1\end{array}\right]$ once.

$$
\left[\begin{array}{lll}
4 & 3 & 1
\end{array}\right] \rightarrow\left[\begin{array}{ll}
4 & 3
\end{array}\right] \oplus\left[\begin{array}{lll}
4 & 2 & 1
\end{array}\right] \oplus\left[\begin{array}{lll}
3 & 3 & 1
\end{array}\right]
$$

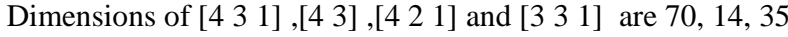
and 21 respectively.

Dimension $(\operatorname{dim})$ of $\left[\begin{array}{lll}4 & 3 & 1\end{array}\right]=\operatorname{dim}$ of [ $\left[\begin{array}{ll}4 & 3\end{array}\right]+\operatorname{dim}$ of $\left[\begin{array}{lll}4 & 2 & 1\end{array}\right]+$ $\operatorname{dim}$ of $\left[\begin{array}{lll}3 & 3 & 1\end{array}\right]$

In general, we have 
$\left[\begin{array}{llll}v_{1} & v_{2} & \ldots v_{i} \ldots v_{n}\end{array}\right]=\sum_{i=n}^{1}\left[\begin{array}{llll}v_{1} & v_{2} & \ldots v_{i-1} \ldots v_{n}\end{array}\right]$

The summation in Eq.(1) indicates that $v_{i}-1 \geq 0$ and $v_{i}-1>v_{i+1}$ in order that $\left[v^{\prime}\right]$ is a young diagram. The dimensions of the both sides of Eq.(1) must be equal; therefore

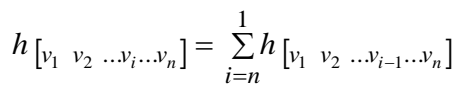

Eq.(1) is referred to as the branching law for the permutation group. The

$S_{n} \supset S_{n-1} \supset S_{n-2} \ldots \supset S_{2} \supset S_{1}$

irreducible basis is the standard basis of the permutation group. It is also called the Young-Yamanouchi basis from the branching law [Eq.(1)] and the fact that the group $S_{2}$ is commutative, we know that the group chain

$$
S_{n} \supset S_{n-1} \supset S_{n-2} \ldots \supset S_{2} \supset S_{1}
$$

is canonical (the last group is redundant).

A Young tableau is an arrangement of the numbers 1, 2, 3, $\ldots, \mathrm{n}$ in a Young diagram in which numbers increase as one moves to the right and as one goes down. A Young tableau is denoted by $Y_{m}^{[v]}$, where $\mathrm{m}$ is the index of the tableau. From a given Young tableau $Y_{m}^{[v]}$, one obtains another Young tableau $Y_{m^{\prime}}^{\left[v^{\prime}\right]}$ involving n-1 numbers by removing the box containing the numbers $\mathrm{n}$; by removing the box with numbers (n-1) one obtains yet another Young tableau $Y_{m^{\prime \prime}}^{\left[v^{\prime \prime}\right]}$ and so on.

\section{CSCO-I OF SYMMETRIC GROUP}

\section{Class operators:}

A sum of all elements belonging to the same class is called class operator. Suppose the $i^{\text {th }}$ class $g_{i}$ elements $a_{1}, a_{2}, a_{3} \ldots a$ $\mathrm{g}_{\mathrm{i}}$, the class operator $\mathrm{C}_{\mathrm{i}}$ is

$$
C_{i}=\sum_{l=1}^{g_{i}} a_{l}{ }^{(i)}, \quad i=1,2 \ldots \ldots . n
$$

Properties:

1. The class operators commute with any elements of the group.

2. The class operators commute with each other

3. The class operators are closed under group multiplication.

Because of the above three properties, the class operator plays a decisive role in the new approach to group representation theory.

\section{Class space:}

The n-dimensional vector space spanned by the n-class operators $\mathrm{C}_{\mathrm{i}}$ of a group is called the class space.

For Example, the three class operators of symmetric group of degree $3\left(S_{3}\right)$ are

$\mathrm{C}_{1}=\mathrm{e}, \quad \mathrm{C}_{2}=\left(\begin{array}{ll}1 & 2\end{array}\right)+\left(\begin{array}{ll}1 & 3\end{array}\right)+\left(\begin{array}{ll}2 & 3\end{array}\right)$ and $\quad \mathrm{C}_{3}=\left(\begin{array}{lll}1 & 2 & 3\end{array}\right)+\left(\begin{array}{lll}1 & 3 & 2\end{array}\right)$
The first kind of complete set of commuting operators of group G (CSCO-I) : If a set of operators $C=\left\{C_{i_{1}}, C_{i_{2}}, \ldots, C_{i_{l}}\right\}$ selected out of the class operators is a CSCO of the class space, then $\mathrm{C}$ is called a CSCO of the first kind of the group or CSCO-I

Note:

1. The set of $n$ class operators $\left(C_{1}, C_{2}, \ldots, C_{n}\right)$ is necessarily a $\mathrm{CSCO}$ of $\mathrm{G}$

2. Any class operator $C_{i}$ of a group $G$ is a function of the CSCO of G.

3. Any eigen vector of the CSCO of group G is necessarily a simultaneous eigen vector of all the $\mathrm{n}$ class operators of group G.

4. Different CSCO's of G are equivalent.

Multiplication table of the class operators of $\mathrm{S}_{3}$ are presented in Table -I. Using the Table - I, the natural representation of the class operators of $S_{3}$ are

$D\left(C_{1}\right)=\left(\begin{array}{lll}1 & 0 & 0 \\ 0 & 1 & 0 \\ 0 & 0 & 1\end{array}\right), D\left(C_{2}\right)=\left(\begin{array}{lll}0 & 3 & 0 \\ 1 & 0 & 2 \\ 0 & 3 & 0\end{array}\right), D\left(C_{3}\right)=\left(\begin{array}{lll}0 & 0 & 2 \\ 0 & 2 & 0 \\ 1 & 0 & 1\end{array}\right)$

Table-I: Multiplication table of the class operators of $S$

\begin{tabular}{|l|l|l|l|}
\hline & $\mathrm{C}_{1}$ & $\mathrm{C}_{2}$ & $\mathrm{C}_{3}$ \\
\hline $\mathrm{C}_{1}$ & $\mathrm{C}_{1}$ & $\mathrm{C}_{2}$ & $\mathrm{C}_{3}$ \\
\hline $\mathrm{C}_{2}$ & $\mathrm{C}_{2}$ & $3\left(\mathrm{C}_{1}+\mathrm{C}_{3}\right)$ & $2 \mathrm{C}_{2}$ \\
\hline $\mathrm{C}_{3}$ & $\mathrm{C}_{3}$ & $2 \mathrm{C}_{2}$ & $2 \mathrm{C}_{1}+\mathrm{C}_{3}$ \\
\hline
\end{tabular}

\section{A NEW GRAPHICAL METHOD TO} INDEX AND RETRIEVE THE SYT

We apply the general frame work outlined in section 2 of Wilf's paper [4] for indexing and retrieving of combinational items.

\section{Problem statement:}

a) Indexing Problem :

Given a SYT in the SYT $([\lambda], \mathrm{n})$, find the index ' $r$ ' such that $0 \leq \mathrm{r} \leq \mathrm{t}-1$, where ' $\mathrm{t}$ ' is the number of SYT in the set $([\lambda], \mathrm{n})$.

b) Retrieving Problem:

Given an integer ' $r$ ': $(0 \leq r \leq t-1)$. Find the corresponding SYT in the SYT ([ $\lambda], \mathrm{n})$.

\section{Index and Retrieving of SYT:}

Steps for Indexing SYT

Step 1: Construct the Graph ' $G$ ' to get the code word for the given SYT

Step 2: For each of the vertices in the codeword, compute the function $f(e)=\sum b\left(e^{\prime}\right)$ (all $e^{\prime}<e$ in the level.)

Step 3: The index of the given SYT $=\sum f(e)$ (for all ' $\mathrm{e}$ ' of the codeword).

Steps for Retrieving SYT

Given an index I of the SYT, get the SYT

Step 1: From the first row in the graph, find the vertex $e_{1}$, where $f\left(e_{1}^{\prime}\right)<I<f\left(e_{1}\right)$ and $e^{\prime}$ is the previous node of $e_{1}$. 
Step 2: Find $I^{\prime}=I-f\left(e^{\prime}\right)$.

Step 3: Repeat step 1 for $I^{\prime}$ in second row and get $e_{2}$.Repeat this step till we reach the terminal node.

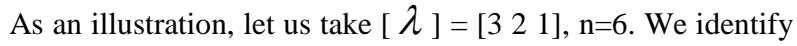
this vertex ' $v$ ' of directed graph as explained in section 2 of ref. [4]. To apply the general framework by Wilf, we first need to identify the branching rules for SYT.

SYT's of symmetric group are equivalent to CSCO-I of symmetric group [5].

Using the branching theorem [5], SYT's can be partitioned as follows.

1. SYT having this group is shown in Figure 1. Note that these are SYT $([3,2,1], 6)$

2. The group that have symbol 6 only once are in Figure 2. We can see that the symbol 6 can be in the first or second or third row, the generic patterns for these are also shown in Figure 2.

The letter $\mathrm{x}$ is where we would have symbol ' 6 '.

The corresponding SYT sets are as follows SYT ([3 2],5), SYT([3,1,1],5), SYT([2 2 1],5)

The sets are 'ordered'. The ordering is defined as the set SYT $\left([\lambda], \mathrm{n}_{1}\right)$ precedes the set

SYT
([ $\left.\bar{\lambda}], \mathrm{n}_{1}\right)$ if $\tilde{\lambda}_{1}>\lambda_{1}$; when $\lambda_{1}=\tilde{\lambda}_{1}$ then $\lambda_{2}=\tilde{\lambda}_{2}$ etc. we shall refer to this definition as definition (1).

3. SYT's having the symbol 5 only once these cases along with theirs generic patterns are shown in Figure 3. The letter $\mathrm{x}$ is where we would have symbol ' 5 '. The sets are 'ordered'. The ordering with definition (1).

4. SYT's having the symbol 4 only once this cases along with their generic patterns are shown in Figure 4.The letter $\mathrm{x}$ is where we would have symbol '4'.The corresponding SYT sets are as follows SYT ([3], 3),SYT([2,1],3), SYT([1 111$], 3)$

5. SYT's having the symbol 3 only once this cases along with their generic patterns are shown in Figure 5.The letter $\mathrm{x}$ is where we would have symbol ' 3 '. The corresponding SYT sets are as follows SYT([2 ],2), SYT([1 1],2)

6. SYT's having the symbol 2 only once this cases along with their generic patterns are shown in Figure 6.The letter $\mathrm{x}$ is where we would have symbol ' 2 '.

The corresponding SYT set is as follows SYT([1],1)

The corresponding SYT sets are as follows SYT ([3 1], 4),SYT ([2 2], 4),SYT ([2 11 1], 4)

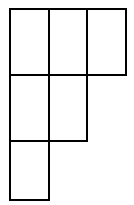

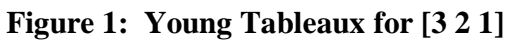
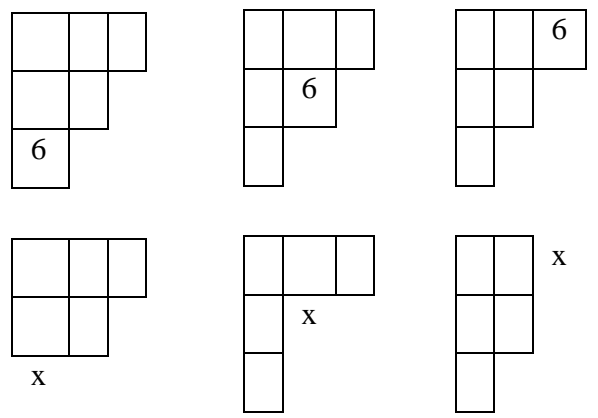

Figure 2: General pattern having symbol ' 6 ' only once
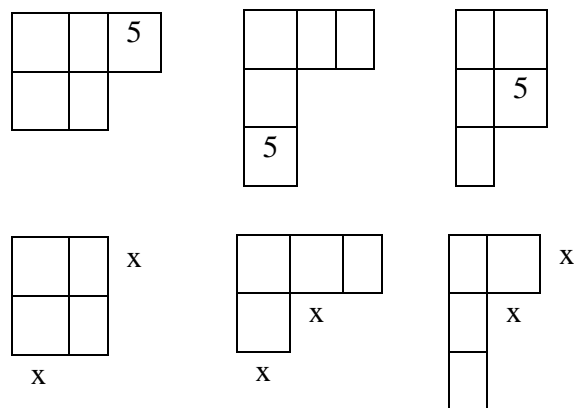

Figure 3: General pattern having symbol ' 5 ' only once. 

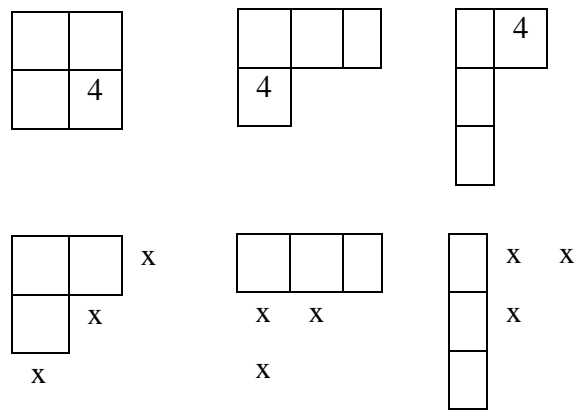

Figure 4 : General pattern having symbol '4' only once
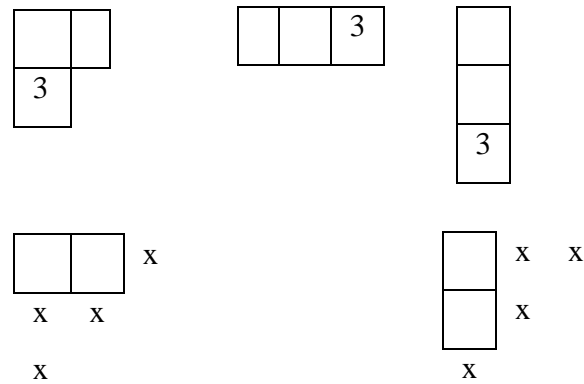

$\mathrm{X}$

Figure 5: General pattern having symbol ' 3 ' only once
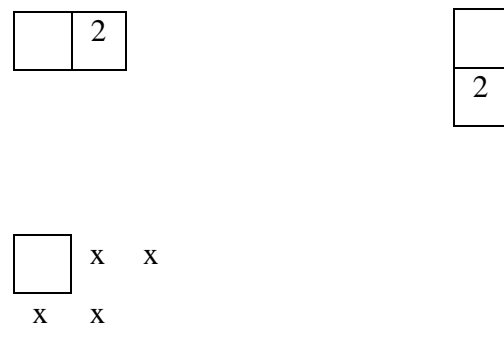

$\mathrm{x}$

Figure 6: General pattern having symbol ' 2 ' only once

The number of SYT's in each of these sets can be calculated using the equation in step 3 . From the above analysis, we get the first level vertices $v_{2}, v_{3}, v_{4}$ the vertex $v$ in the directed graph $\mathrm{G} \quad$ (section 2 in reference [4])

Let $\mathrm{b}(v)$ be the number of SYT's under $v$. This is given by the equation in step 3 , noting that $v=$ SYT's ([3 2 1], 6).

The corresponding steps are given as follows:

Compute the product of hook lengths [5] for the partition [3 $21]=$

$$
\frac{6 !}{5 \cdot 3 \cdot 3}=\frac{6 \cdot 5 \cdot 4 \cdot 3 \cdot 2 \cdot 1}{5 \cdot 3 \cdot 3}=16
$$

Therefore $\mathrm{b}(v)=$ the number of SYT's $=16$.

Similarly $\mathrm{b}\left(v_{1}\right), \mathrm{b}\left(v_{2}\right) \ldots$ are calculated. The result is shown in Table II. The directed graph $\mathrm{G}$ derived based on the discussion in section 2 of reference [4] is shown in Figure 7 (combined with Table III). We note that in the directed graph $\mathrm{G}$ in Figure 7. The vertex $v$ is the combinatorial object of order $v$ for SYT ([ $\left.\left.\begin{array}{lll}3 & 2 & 1\end{array}\right], 6\right)$. Each path from vertex to the terminal vertex $\tau$ is called 'code word 'as in reference [4]. Each edge in the code word connects a vertex at a certain level to another vertex in its immediate lower level. In the present example between the vertex $v$ and terminal vertex $\tau$, we have 5 levels in the graph in Figure 7, the vertices $v_{2}, v_{3}$ ,$v_{4}$ corresponding to the first level the vertices $v_{5}, v_{6}, v_{7}$ corresponding to the second level the vertices $v_{8}, v_{9}, v_{10}$ corresponding to the third level the vertices $v_{11}, v_{12}$ corresponding to the forth level. The vertex $v_{13}$ corresponding to fifth level .The details of the connection between the vertices of different level is given in Table III. 


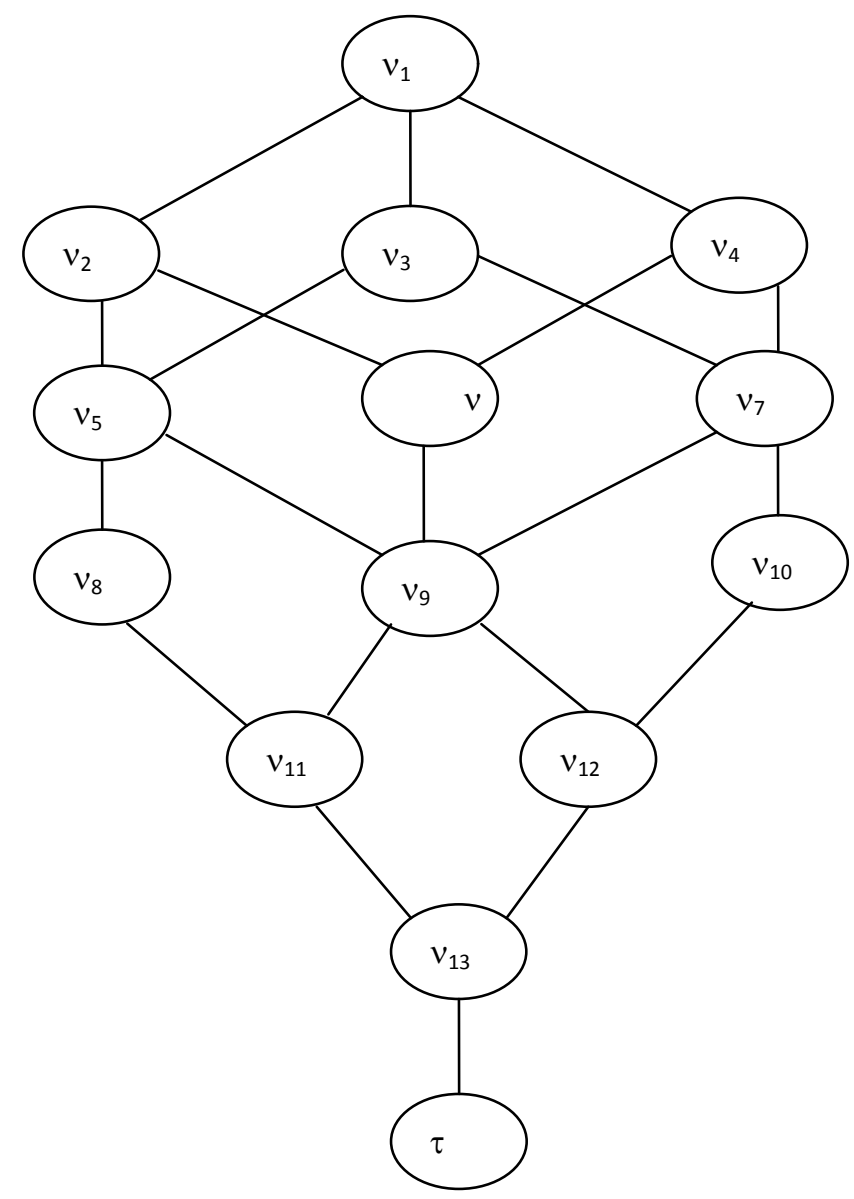

Figure 7: Graphical representation

Table II Values of $v$ and $b(v)$

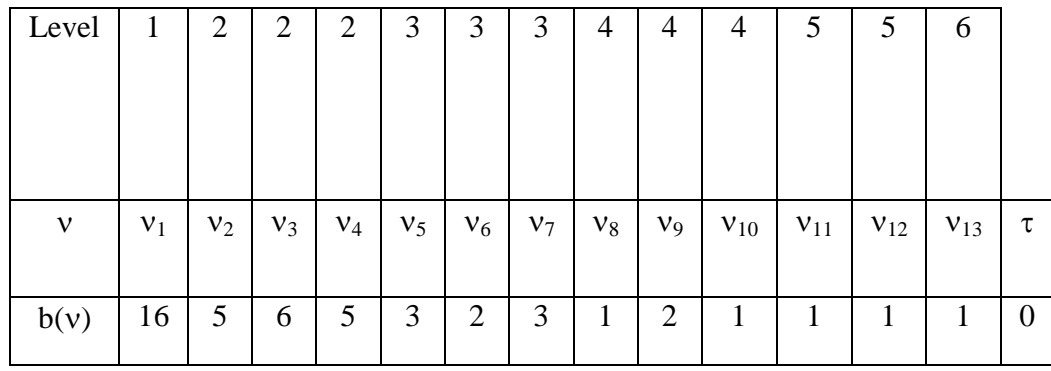

In the graph $\mathrm{G}$, the numbers under each vertex are the number of SYT's for that vertex or the number of 'code words' from the vertex. This number is obtained by using the equation in the text book [5]. We could also note that this number of 'code words' of a vertex is the sum of 'code words' of the 'final vertices' of the vertex.

For example from Table III, we note that the vertex $v_{3} \rightarrow\left(v_{5}, v_{7}\right)$.

This implies that $b\left(v_{3}\right)=b\left(v_{5}\right)+b\left(v_{7}\right)$.
The SYT corresponding to the 'code word' is $v \rightarrow v_{2} \rightarrow v_{5} \rightarrow v_{9} \rightarrow v_{12} \rightarrow v_{13} \rightarrow \tau$ is the figure 8. In the graph, each 'code word' uniquely represents a SYT for ([3 2 1], $\mathrm{n}=6$ ).As an illustration the 'code word' $v \rightarrow v_{2} \rightarrow v_{5} \rightarrow v_{9} \rightarrow v_{12} \rightarrow v_{13} \rightarrow \tau$ corresponds to the SYTs shown in figure 8.In the graph ' $\mathrm{G}$ ', we depict the pair $(v, b(v))$ inside a node. The vertex is identified by the number and corresponding $b(v)$ appears just under it. The table-II gives the values of $b(v)$, for each vertex ' $v$ ' of the graph ' $\mathrm{G}$ ' 
Table III: Interconnection of nodes

\begin{tabular}{|c|c|c|}
\hline$v_{1} \rightarrow\left(v_{2}, v_{3} v_{4}\right)$ & $v_{2} \rightarrow\left(v_{5}, v_{6}\right)$ & $v_{3} \rightarrow\left(v_{5}\right.$, \\
\hline$v_{7} \rightarrow\left(v_{9}, v_{10}\right)$ & $v_{8} \rightarrow\left(v_{11}\right)$ & $v_{9} \rightarrow\left(v_{11}\right.$ \\
\hline
\end{tabular}

Indexing for the SYT, shown in Figure-8 as discussed earlier, the codeword for the SYT is

$v \rightarrow v_{2} \rightarrow v_{5} \rightarrow v_{9} \rightarrow v_{12} \rightarrow v_{13} \rightarrow \tau$

Table-3: Description of nodes $v_{1}$ to $v_{13}$

Description of nodes for first level

$v_{1}=\operatorname{SYT}\left(\left[\begin{array}{lll}3 & 2 & 1\end{array}\right], 6\right)$

Description of nodes for second level

$$
\begin{aligned}
& v_{2}=\operatorname{SYT}\left(\left[\begin{array}{ll}
3 & 2
\end{array}\right], 5\right) \quad v_{3}=\operatorname{SYT}\left(\left[\begin{array}{lll}
3 & 1 & 1
\end{array}\right], 5\right) \\
& v_{4}=\operatorname{SYT}\left(\left[\begin{array}{lll}
2 & 2 & 1
\end{array}\right], 5\right)
\end{aligned}
$$

Description of nodes for third level

$$
\begin{aligned}
& v_{5}=\mathrm{SYT}([31], 4) \quad v_{6}=\mathrm{SYT}([2,2], 4) \\
& v_{7}=\operatorname{SYT}\left(\left[\begin{array}{lll}
2 & 1 & 1
\end{array}\right], 4\right)
\end{aligned}
$$

Description of nodes for fourth level

$$
\begin{aligned}
& v_{8}=\operatorname{SYT}([3], 3) \\
& v_{10}=\operatorname{SYT}\left(\left[\begin{array}{lll}
1 & 1 & 1
\end{array}\right], 3\right)
\end{aligned}
$$

Description of nodes for fifth level

$$
v_{11}=\mathrm{SYT}([2], 2) \quad v_{12}=\mathrm{SYT}\left(\left[\begin{array}{ll}
1 & 1], 2)
\end{array}\right.\right.
$$

Description of nodes for sixth level

$$
v_{13}=\mathrm{SYT}([1], 1)
$$

\begin{tabular}{|l|l|l|}
\hline 1 & 3 & 4 \\
\hline 2 & 5 & \\
\cline { 1 - 2 } 6 & \multicolumn{2}{|c}{} \\
\cline { 1 - 1 } & &
\end{tabular}

Figure 8: The SYT of G

(Steps for ranking) and their entries in table-III this gives us

$$
\begin{array}{lc}
f\left(v_{2}\right)=b\left(v_{5}\right)+b\left(v_{6}\right) & f\left(v_{5}\right)=b\left(v_{8}\right)+b\left(v_{9}\right) \\
f\left(v_{9}\right)=b\left(v_{11}\right)+b\left(v_{12}\right) & f\left(v_{12}\right)=b\left(v_{12}\right) \\
f\left(v_{13}\right)=b\left(v_{13}\right) &
\end{array}
$$

Using the values of $\mathrm{b}(\mathrm{)}) \mathrm{s}$ in table II, we get
We take $v_{5}$ as root. Use relation $v_{5} \rightarrow\left(v_{8}, v_{9} ..\right)$ Repeating steps 1 and 2, we get the following:

$$
v_{5} \rightarrow v_{9}, v_{9} \rightarrow v_{12}, v_{12} \rightarrow v_{13} \text {. }
$$

Hence, the codeword corresponding to the index $=12$ is $v \rightarrow v_{2} \rightarrow v_{5} \rightarrow v_{9} \rightarrow v_{12} \rightarrow v_{13} \rightarrow \tau$. This corresponds to the SYT in figure 8 .

\section{CONCLUSION}

In this paper, we have proposed a method to index and retrieve SYTs. This has been achieved by using the general framework of Wilf and the properties of SYT. A new graphical method to index and retrieve the Standard Young Tableau for a partition of degree $n$ in $S_{n}$ has presented. This method has been illustrated with an example using a partition of degree 6 in symmetric group $S_{6}$.

\section{REFERENCES}

[1] Stanley, R. P. (1980). The character generator of SU (n). Journal of Mathematical Physics, 21(9), 2321-2326.

[2] Patera, J. (2004). RT Sharp and Generating Functions in Group Theory. Symmetry in Physics: In Memory of Robert T. Sharp, 34, 159.

[3] Delaney, R. M., \& Gruber, B. (1969). Inner and restriction multiplicity for classical groups. Journal of Mathematical Physics, 10(2), 252-265.

[4] Wilf, H. S. (1977). A unified setting for sequencing, ranking, and selection algorithms for combinatorial objects. Advances in Mathematics, 24(3), 281-291

[5] Chen J Q, Group Representation theory for physicists, World Scientific publishing Co. Pvt.Ltd., 1989

[6] Shankar, N. R., Suryanarayana, C., Sekhar, S. R., \& Rao, A. G. (2010). The Kronecker Product of Symmetric Group Representations Using Schur Functions. International Journal of Algebra, 4(12), 579-584. 